\title{
Concurrent Treatment with Prolonged Exposure for Co-Occurring Full or Subthreshold Posttraumatic Stress Disorder and Substance Use Disorders: A Randomized Clinical Trial
}

\author{
Lesia M. Ruglass $^{\text {a }}$ Teresa Lopez-Castro $^{a} \quad$ Santiago Papini ${ }^{b} \quad$ Therese Killeen $^{c}$ \\ Sudie E. Back ${ }^{c}$ Denise A. Hien ${ }^{d}$ \\ ${ }^{a}$ The City College of New York of CUNY, New York, NY, ${ }^{b}$ The University of Texas at Austin, Austin, TX, ${ }^{c}$ Medical \\ University of South Carolina, Charleston, SC, and d Gordon F. Derner Institute for Advanced Psychological Studies, \\ Adelphi University and Department of Psychiatry, Columbia University College of Physicians and Surgeons, \\ New York, NY, USA
}

\section{Keywords}

Posttraumatic stress disorder - Substance use disorder . Comorbidity · Integrated treatment $\cdot$ Randomized clinical trial

\begin{abstract}
Background: To test whether an integrated prolonged exposure (PE) approach could address posttraumatic stress disorder (PTSD) symptoms effectively in individuals with cooccurring substance use disorders (SUD), we compared concurrent treatment of PTSD and SUD using PE (COPE) to relapse prevention therapy (RPT) for SUD and an active monitoring control group (AMCG). Methods: We conducted a randomized 12-week trial with participants ( $n=110 ; 64 \%$ males; 59\% African Americans) who met Diagnostic and Statistical Manual of Mental Disorders, fourth edition, text revision criteria for full or subthreshold PTSD and SUD. Participants were randomly assigned to COPE $(n=39), \operatorname{RPT}(n=43)$, or AMCG $(n=28)$. Results: At the end-of-treatment, COPE and RPT demonstrated greater reduction in PTSD symptom severity relative to AMCG (COPE-AMCG $=-34.06, p<0.001$;
\end{abstract}

\section{KARGER}

(C) 2017 S. Karger AG, Basel

E-Mail karger@karger.com

www.karger.com/pps
RPT-AMCG $=-22.58, p=0.002)$. Although the difference between COPE and RPT was not significant in the complete sample, the subset of participants with full (vs. subthreshold) PTSD demonstrated significantly greater reduction of PTSD severity in COPE relative to RPT. Both treatments were superior to $A M C G$ in reducing the days of primary substance use (COPE-AMCG $=-0.97, p=0.01$; RPT-AMCG $=-2.07, p<0.001$ ). Relative to COPE, RPT showed significantly more improvement in SUD outcome at end-of-treatment (RPT-COPE = $-1.10, p=0.047)$. At 3-month follow-up, COPE and RPT maintained their treatment gains and were not significantly different in PTSD severity or days of primary substance use. Conclusion: COPE and RPT reduced PTSD and SUD severity in participants with PTSD + SUD. Findings suggest that among those with full PTSD, COPE improves PTSD symptoms more than a SUD-only treatment. The use of PE for PTSD was associated with significant decreases in PTSD symptoms without worsening of substance use.

(c) 2017 S. Karger AG, Basel

Clinical Trials Registration - clinicaltrials.gov identifier: NCT01365247.

Lesia M. Ruglass, $\mathrm{PhD}$

Department of Psychology, The City College of New York of CUNY

160 Convent Avenue, NAC Building, Rm 7/120

New York, NY 10031 (USA)

E-Mail ruglass.ccny@gmail.com 
Epidemiological and clinical research have consistently documented a strong association between posttraumatic stress disorder (PTSD) and substance use disorders (SUD). The prevalence rate of SUD among those with PTSD is high, with estimates ranging from 21 to $43 \%$ [ 1 , 2]. Conversely, among individuals receiving SUD treatment, the prevalence of PTSD ranges from 26 to $43 \%$ [3, 4]. Compared to individuals with only one disorder, individuals with co-occurring PTSD and SUD (PTSD + SUD) exhibit more severe symptomatology and comorbidities, greater functional impairments, poorer treatment outcomes, and heightened vulnerability to relapse [5-7]. The societal costs of PTSD + SUD are tremendous, necessitating effective treatment approaches designed for this population.

While the evidence base for the treatment of either PTSD or SUD alone is robust [8-11], there is less consensus regarding how best to treat PTSD + SUD $[5,12,13]$. Although the efficacy of prolonged exposure (PE) therapy [14] for PTSD is well-established [15], its tolerability among patients with co-occurring SUD has been questioned $[16,17]$ with anecdotal concerns that substancerelated cognitive impairments are a barrier to imagery procedures, or that the intervention would be too emotionally distressing, triggering a relapse or increasing treatment attrition. As a result, most PE trials have excluded individuals with SUD, thus limiting the evidence of its efficaciousness among the significant subpopulation of individuals with PTSD + SUD.

Despite these ongoing concerns, support for the use of exposure-based treatments among PTSD + SUD populations is building $[3,18-21]$. In its first randomized controlled trial, concurrent treatment of PTSD and SUD using $\mathrm{PE}$ (COPE), an integration of relapse prevention therapy (RPT) for substance misuse and PE for PTSD, led to enhanced reduction of PTSD severity with no increase in substance dependence severity compared to usual treatment [3]. In a four-arm trial comparing PE with naltrexone or placebo to supportive counseling with naltrexone or placebo, significant reductions in PTSD and alcohol use were observed across all groups [20]. While PE did not demonstrate a relative advantage at end-of-treatment, PE with naltrexone was associated with lower likelihood of relapse at 6 month follow-up [20]. In a third trial, an integrated exposure-based cognitive behavioral therapy for comorbid PTSD and alcohol use disorders (AUD) showed significantly greater reductions in PTSD and AUD compared to cognitive behavioral therapy for AUD plus supportive counseling [21]. Moreover, integrated treatment participants who completed one or

COPE for Co-Occurring PTSD and SUD more exposure sessions were twice as likely to experience clinically significant changes in PTSD severity as those in the AUD-only intervention [21].

As the second randomized trial of COPE, we sought to extend prior findings [3] by delivering COPE to a racially/ ethnically diverse, polysubstance-dependent sample and comparing its efficacy relative to the gold standard treatment for SUD alone [22], RPT, and an active monitoring control group (AMCG). Based on previous research, our primary aim was to test whether COPE and RPT would show significantly greater reductions in PTSD and SUD symptom severity than AMCG; and our secondary aim was to test whether COPE would be superior to RPT on PTSD and SUD outcomes.

\section{Method}

\section{Recruitment}

Participants were recruited through advertisements and outpatient referrals in New York City between September 2008 and January 2014 and provided written informed consent prior to baseline assessment. Inclusion criteria were: (1) Diagnostic and Statistical Manual of Mental Disorders, fourth edition, text revision (DSMIV-TR) [23] criteria for full PTSD, or subthreshold PTSD defined as meeting criterion $\mathrm{A}, \mathrm{B}$, either $\mathrm{C}$ or D, and $\mathrm{E}$ and $\mathrm{F}$ [24]. We included individuals with subthreshold PTSD as studies indicate they have similar levels of distress, impairment, and psychiatric comorbidity as those with full PTSD and thus comprise a clinically meaningful diagnostic group [25-27]; and (2) DSM-IV-TR criteria for either past or current alcohol or substance dependence and alcohol/ substance use in the prior 90 days. Given the chronic, relapsing nature of SUD in the context of co-occurring mental health disorders $[28,29]$, we included individuals with past dependence who were currently using substances. Exclusion criteria were: (1) psychotic, schizoaffective or bipolar disorder; (2) current severe depression (indicated by Beck Depression Inventory score $\geq 30$ ) or suicide risk; (3) currently in an abusive relationship; (4) concurrent participation in PTSD-specific treatment; (5) start or regimen change of any psychotropic medication 8 weeks before study participation; (6) organic mental syndrome. The Institutional Review Board of the City College of New York approved all procedures.

\section{Randomization}

Randomization was stratified by sex, baseline severity of substance and alcohol dependence (high or low operationalized from median split of Addiction Severity Index [ASI] Lite composite scores) and PTSD severity (high or low defined by the cutoff score of 60 on the Clinician-Administered PTSD Scale [CAPS]). Urn randomization procedures were employed to balance these factors across groups. One year prior to study completion, randomization to the AMCG group was halted $(n=28)$ to increase the sample sizes of the two active treatment groups. An independent biostatistician conducted the randomization allocation. A research coordinator revealed group allocation to participants after they provided informed consent. All research assessors were blind to group allocation. 


\section{Interventions}

COPE integrates empirically supported models of PE for PTSD $[14,30]$ and RPT for SUD $[22,31]$. Sessions $1-3$ focus on goalsetting, psychoeducation, and cognitive-behavioral strategies. To address behavioral avoidance and fear associated with trauma memories, in vivo and imaginal exposures begin in sessions 4 and 5 , respectively, and continue until session 11 . In vivo exercises are selected from a collaboratively constructed hierarchy of safe, yet avoided situations. Participants engage in prolonged contact with feared situations until subjective distress is significantly reduced. During imaginal exposures, participants repeatedly recount a traumatic memory with emphasis on accessing related thoughts, emotions, and physical sensations for approximately $30-45$ min during session. Imaginal narratives are audio-recorded for daily listening between sessions. Relapse prevention strategies were integrated with PE during each 90-min session. The integrated RPT components explicitly targeted PTSD-related triggers for substance use. Participants recorded the progress of exposure exercises, substance use cravings, and use of coping skills.

RPT $[22,31]$ is a cognitive-behavioral SUD intervention that focuses on coping strategies to effectively manage situations that increase the risk of substance use relapse. These sessions did not explicitly target trauma or PTSD symptomatology. Psychoeducation, role plays, and active problem-solving exercises are combined with at-home assignments and geared towards increasing participants' self-efficacy in preventing relapse. RPT sessions were also 90-min in duration to control for session length.

In contrast to traditional waitlist control groups, where there is minimal contact, AMCG participants met weekly with research assistants over a 12-week period to complete self-report measures, urine toxicology, alcohol breathalyzer, and confirm general health/ safety. AMCG participants did not receive any interventions targeting PTSD, SUD, or both. After completion of a 12-week waiting period, AMCG participants were given outpatient treatment referrals or the opportunity to enroll in one of the study's 2 active treatments.

Both COPE and RPT treatments consisted of 12 individual weekly sessions lasting $90 \mathrm{~min}$. Participants had up to 14 weeks for treatment completion. Treatments were delivered by $\mathrm{PhD}(n=7)$ or master's level $(n=3)$ clinicians who received $2 \frac{1}{2}$ days of training, including review of intervention-specific theory and techniques, manual review, and practice within trainer- and traineeconducted mock intervention sessions. Clinicians received 1 -h weekly case supervision for the duration of the study. All therapy sessions were audiotaped, and randomly selected sessions (approximately, $25 \%$ for each therapist) were rated by one of the developers of COPE using adherence and competency measures adapted from previous studies $[19,32]$. An example of one such rating question is "To what extent and level of skill did the therapist conduct the imaginal exposure procedure (beginning and end point of trauma narrative, repetitions, SUDS ratings throughout exposure, timeframe, present tense, eyes closed, safe environment, minimal interaction, etc.)?" The rating scales ranged from $0=$ not at all to $5=$ excellent/extensive adherence and competence. Throughout the study, therapists met weekly with the therapy fidelity rater for supervision. If adherence fell below competency criterion ( $<3$ on a scale of $1-5)$, additional supervision was provided. The same therapists delivered the CBT relapse prevention intervention. No therapist was withdrawn from the study because of inadequate adherence or competency in the delivery of both manualized therapies (adequate $=3$ in a scale of $1-5$ ).

\section{Data Collection}

Research assistants collected urine samples for drug toxicology, alcohol breathalyzer readings, and self-report measures during every visit. Blind independent assessors interviewed participants at baseline, end-of-treatment, and follow-ups. Only individuals in RPT or COPE completed assessments at 1-, 2-, and 3-months follow-up.

\section{Measures}

Age, sex, race/ethnicity, education, employment pattern, and income were collected during the baseline interview. The Structured Clinical Interview for DSM-IV for Axis I Disorders (SCID-I) [33] was used to assess SUD diagnoses and age of onset, as well as the presence of other lifetime anxiety, mood, or psychotic disorders.

The CAPS [34] was used at baseline and follow-ups to measure PTSD symptom severity in the previous 30 days, and the modified PTSD Symptom Scale Self-Report (MPSS-SR) [35] was used during weekly visits to assess self-reported symptom severity in the previous 7 days. Although the instruments have different ranges, both yield a total score comprised of the sum of frequency and intensity ratings of each of the 17 DSM-IV-TR PTSD symptoms. Psychometric studies of the MPSS-SR with similar PTSD + SUD treatment samples demonstrate its high concurrent validity with the CAPS, and suggest that it is a reliable tool for monitoring PTSD symptoms [36].

Primary SUD diagnosis was based on the number of dependence criteria from the SCID. The frequency of primary substance use in the past 30 days was assessed at baseline and follow-ups using the ASI Lite [37]. During treatment, the Substance Use Inventory (SUI) [38] was administered weekly to collect self-reported days of primary substance use.

\section{Sample Size}

Sample size calculations were made for the primary analysis assessing differences across the three groups in MPSS-SR change from pre-randomization to end-of-treatment. A sample size of 50 per group was needed to detect a medium effect size $(d=0.5)$ between treatment conditions, with a 2 -sided significance level of 0.05 , a power of $80 \%$, and repeated measures correlation of 0.6 [39]. Due to lower than anticipated recruitment, the study had approximately $70 \%$ power to detect an effect of $d=0.5$, or $80 \%$ power to detect an effect of $d=0.56$. High non-completion rates, which are especially common in PTSD + SUD treatment trials [19, 40, 41], also bear on the power to detect differences at end-of-treatment.

\section{Statistical Analyses}

Baseline differences were explored with $\chi^{2}$ tests for categorical variables, one-way ANOVAs for continuous variables, and nonparametric Kruskal-Wallis H-tests for non-normal count data. Following the intent-to-treat principle, generalized linear mixed models of treatment outcomes included all participants. Missing data were estimated using all available data in each model; therefore, imputation was not necessary. Models were specified to the distribution that best fit each outcome (i.e., normal distribution for MPSS-SR, CAPS, and previous 30 days of substance use and Poisson-distribution for previous 7 days of substance use). Results and 95\% CIs are reported in original units.

Models included fixed effects of group, time, and a group-bytime interaction term, as well as random intercepts. The mean 
change through end-of-treatment was examined by comparing the three groups on weekly (baseline through the last session of treatment) self-reported PTSD symptoms (MPSS-SR total score) and days of primary substance use (SUI). Self-report measures were used for the three group comparisons as the time frame of assessment was weekly. Within- and between-group differences in change from baseline to the end-of-treatment session were assessed.

Since the AMCG participants were provided with referrals or given the opportunity to enroll in active treatment after end-oftreatment assessment, only the active treatments were assessed beyond the end-of-treatment; therefore, the duration of treatment effects at the follow-ups were assessed in separate models. For these analyses, PTSD outcome was measured with past 30 days CAPS total severity score and primary SUD was measured with past 30 days of use reported in the ASI since the time frame between assessments was monthly. In addition to planned withingroup contrasts between baseline and the 1- and 3-month followups (to determine the level of reduction after treatment), the 3 -month follow-up was contrasted with the 1-month follow-up to assess the duration of treatment effect. Intermediate assessments were collected at the end-of-treatment (representing severity during last month of treatment) and 2-month follow-up; these were included in the models to increase power and improve missing data estimation.

\section{Results}

\section{Baseline Characteristics}

Participant flow is illustrated in Figure 1. Baseline characteristics of 110 randomized participants are presented in Table 1. No differences were observed in age, education level, sex or race/ethnicity distributions, employment, SUD diagnoses, or comorbid anxiety disorder between the three conditions. All participants met criteria for current alcohol and/or substance dependence except for two in the RPT group who met for past alcohol dependence. Baseline CAPS scores were significantly different, $F(2,107)=3.85, p=0.02$. Although there was no significant difference between treatments (COPE: mean $=55.38$, $\mathrm{SD}=16.40$ vs. $\mathrm{RPT}$ : mean $=57.70, \mathrm{SD}=20.81, p=0.55$ ), the AMCG (mean $=46.39, \mathrm{SD}=11.08$ ) had significantly lower CAPS total scores compared to each of the treatment groups (both $p$ values $<0.04$ ). There was no difference in the proportion of individuals with subthreshold PTSD in COPE $(35.9 \%, n=14)$, RPT $(32.6 \%, n=14)$, and $\operatorname{AMCG}(39.3 \%, n=11), \chi^{2}(2)=0.59, p=0.74$. The AMCG had a significantly lower proportion $(7.1 \%, n=2)$ of individuals diagnosed with current major depressive disorder compared to COPE $(33.3 \%, n=13)$ and RPT $(37.2 \%$, $n=15), \chi^{2}(2)=8.37, p=0.02$. There was a difference in the number of sessions attended among the groups, $F(2,107)=3.20, p=0.045$, driven by the difference be-

COPE for Co-Occurring PTSD and SUD tween COPE and AMCG (COPE: mean $=6.08, \mathrm{SD}=4.75$ vs. AMCG: mean $=8.82, \mathrm{SD}=3.76, p=0.01)$. The mean number of sessions attended was not significantly different between treatments (COPE: mean $=6.08, \mathrm{SD}=4.75$ vs. RPT: mean $=7.21, \mathrm{SD}=4.40, p=0.27)$. However, when included in the outcome models, the number of sessions attended was not a significant covariate and did not significantly alter the outcome of the original analyses (i.e., the group-by-time interactions). One participant from the COPE group was hospitalized after a suicide attempt. Although the event was determined to be nonstudy related and the participant wished to continue treatment, study protocol required that the individual be removed from the active intervention and referred to continued outpatient treatment.

\section{PTSD Outcome}

There was a significant group-by-time interaction $(p<$ 0.001 ) in the model of MPSS-SR total (Fig. 2). Only the active treatments showed significant reductions (i.e., end-of-treatment total minus baseline total) in MPSS-SR total scores by the end-of-treatment session (COPE = $-42.99,95 \%$ CI -56.30 to $-29.68, p<0.001$; RPT $=-31.51$, $95 \%$ CI -40.64 to $-22.38, p<0.001)$. Each active treatment was associated with significantly greater symptom reduction (i.e., the difference in the amount of symptom reduction between groups) than AMCG (COPE-AMCG = $-34.06,95 \%$ CI -51.36 to $-16.75, p<0.001$; RPT$\mathrm{AMCG}=-22.58,95 \% \mathrm{CI}-36.92$ to $-8.24, p=0.002)$. The difference between COPE and RPT was non-significant (COPE-RPT $=-11.48,95 \%$ CI -27.62 to $4.67, p=0.16$ ).

Next, COPE and RPT were compared on CAPS total severity scores through follow-up. Both treatments showed significant reductions in CAPS scores at the 1-month follow-up (COPE $=-27.12,95 \% \mathrm{CI}-35.84$ to $-18.40, p<0.001 ; \mathrm{RPT}=-25.38,95 \% \mathrm{CI}-33.12$ to -17.64 , $p<0.001)$, and 3-month follow-up (COPE $=-28.31,95 \%$ CI -36.01 to $-20.60, p<0.001$; RPT $=-26.71,95 \% \mathrm{CI}$ -34.28 to $-19.14, p<0.001)$ relative to baseline. However, there was no evidence of differential treatment effects, as indicated by the lack of a group-by-time interaction ( $p=$ 0.86), and the lack of between-group differences in CAPS scores at the follow-ups. There were no significant within- or between-group differences in CAPS change from the 1- to the 3-month follow-ups.

Further analyses were conducted to examine whether treatment effects on PTSD outcomes were moderated by baseline PTSD status (full vs. subthreshold). The models included a 3-way interaction of group, PTSD status, and time. In the model of within-treatment change, the 3-way 
397 No show

212 Did not meet inclusion criteria 173 PTSD DX

39 Substance dependence

221 Met exclusion criteria

26 Bipolar DX

22 Suicide risk

17 History of severe violence

156 Other (Psychotic DX,

severely depressed, currently in abusive relationship,

necessitating detoxification and

no interest in treatment)

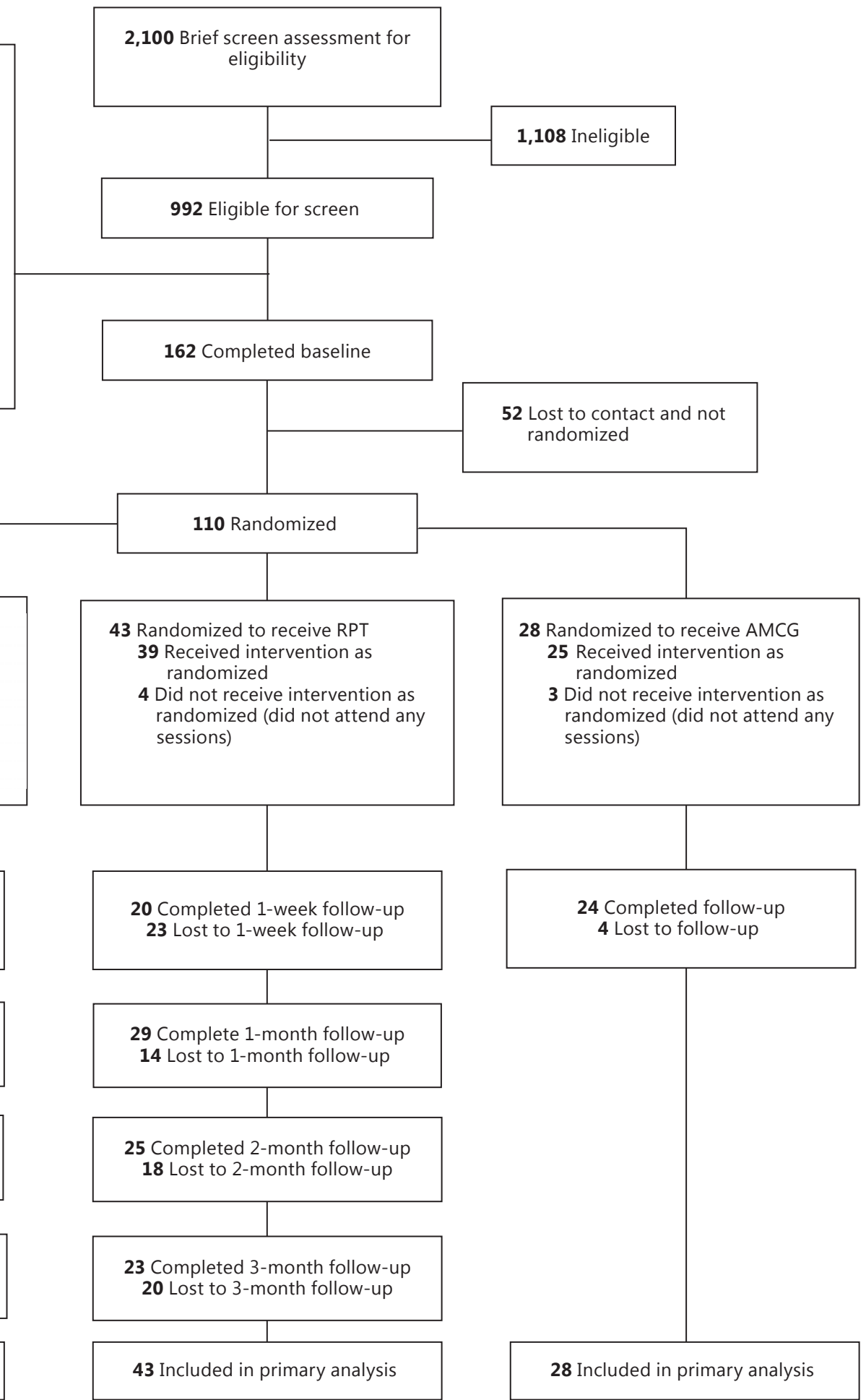

39 Randomized to receive COPE 34 Received intervention as randomized

5 Did not receive intervention as randomized (4 did not attend any sessions; 1 adverse event)

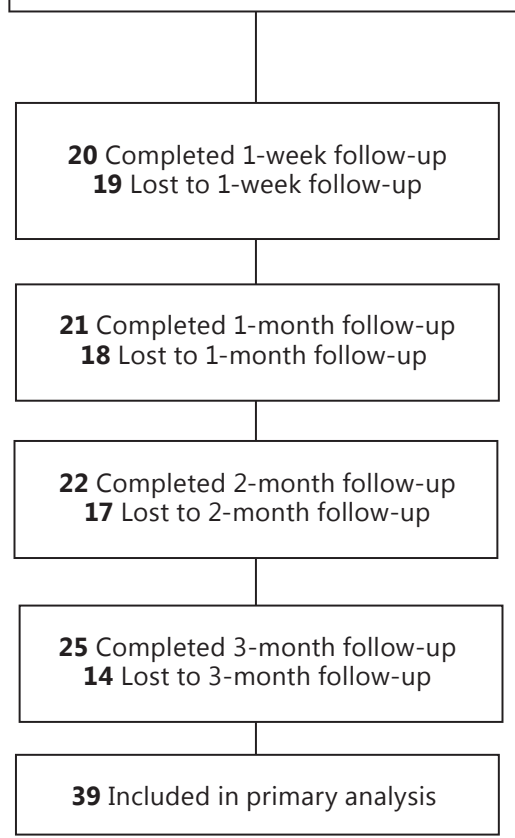

Randomized to receive RPT randomized (did not attend any sessions)

Completed 1-week follow-up 23 Lost to 1-week follow-up

Complete 1-month follow-up 14 Lost to 1-month follow-up

5 Completed 2-month follow-up 18 Lost to 2-month follow-up

3 Completed 3-month follow-up 20 Lost to 3-month follow-up

43 Included in primary analysis
Randomized to receive AMCG randomized (did not attend any essions)

Completed follow-up

Fig. 1. CONSORT diagram of participant flow through the protocol. PTSD, posttraumatic stress disorder; DX, diagnosis; COPE, concurrent treatment of PTSD and substance use disorder using prolonged exposure; RPT, relapse prevention therapy; AMCG, active monitoring control group. 
Table 1. Demographic and baseline clinical characteristics

\begin{tabular}{|c|c|c|c|}
\hline Characteristic & COPE $(n=39)$ & $\operatorname{RPT}(n=43)$ & $\operatorname{AMCG}(n=28)$ \\
\hline \multicolumn{4}{|l|}{ Demographic } \\
\hline Age, years & $43.08 \pm 10.00$ & $44.21 \pm 9.05$ & $47.18 \pm 8.21$ \\
\hline Female & $11(28.2)$ & $16(37.2)$ & $13(46.4)$ \\
\hline \multicolumn{4}{|l|}{ Race/ethnicity } \\
\hline Black/African American & $21(53.8)$ & $28(65.1)$ & $16(57.1)$ \\
\hline Hispanic/Latino & $10(25.6)$ & $9(20.9)$ & $3(10.7)$ \\
\hline White & $6(15.4)$ & $6(14.0)$ & $8(28.6)$ \\
\hline Other & $2(5.1)$ & 0 & $1(3.6)$ \\
\hline \multicolumn{4}{|l|}{ Employment pattern (past 3 years) } \\
\hline Full time & $14(35.9)$ & $8(18.6)$ & $11(39.3)$ \\
\hline Part time/student & $15(38.5)$ & $18(41.9)$ & $9(32.1)$ \\
\hline Unemployed/disability & $10(25.6)$ & $17(39.5)$ & $8(28.6)$ \\
\hline Education, years & $13.31 \pm 1.92$ & $13.13 \pm 2.46$ & $13.21 \pm 3.52$ \\
\hline \multicolumn{4}{|l|}{ Criterion A trauma exposure } \\
\hline \multicolumn{4}{|l|}{ Type } \\
\hline Physical assault & $22(56.4)$ & $28(65.1)$ & $15(53.6)$ \\
\hline Sexual assault & $17(43.6)$ & $17(39.5)$ & $8(28.6)$ \\
\hline Accident or disaster & $1(2.6)$ & $5(11.6)$ & $3(10.7)$ \\
\hline Sudden injury or death of other & $12(30.8)$ & $20(46.5)$ & $14(50.0)$ \\
\hline Other & $6(15.4)$ & $3(7.0)$ & $2(7.1)$ \\
\hline Multiple trauma & $21(53.8)$ & $35(81.4)$ & $21(75.0)$ \\
\hline Age at first trauma & $17.90 \pm 13.64$ & $18.49 \pm 14.13$ & $21.43 \pm 14.42$ \\
\hline Time since last trauma, years & $16.15 \pm 14.98$ & $11.95 \pm 10.73$ & $18.43 \pm 15.56$ \\
\hline \multicolumn{4}{|l|}{ Alcohol and substance use } \\
\hline Alcohol dependence & $30(76.9)$ & $35(81.4)$ & $20(71.4)$ \\
\hline Drug dependence & $25(64.1)$ & $30(69.8)$ & $18(64.3)$ \\
\hline Alcohol and drug dependence & $16(41.0)$ & $24(55.8)$ & $10(35.7)$ \\
\hline \multicolumn{4}{|l|}{ Primary substance } \\
\hline Alcohol & $19(48.7)$ & $18(41.9)$ & $12(42.9)$ \\
\hline Cannabis & $3(7.7)$ & $4(9.3)$ & $2(7.1)$ \\
\hline Cocaine & $6(15.4)$ & $6(14.0)$ & $6(21.4)$ \\
\hline Alcohol and stimulants & $8(20.5)$ & $13(30.2)$ & $6(21.4)$ \\
\hline Other polysubstance & $3(7.7)$ & $2(4.6)$ & $2(7.1)$ \\
\hline \multicolumn{4}{|l|}{ Other diagnoses } \\
\hline Major depressive disorder & $13(33.3)$ & $16(37.2)$ & $2(7.1)$ \\
\hline Anxiety (panic, phobia, SAD, or GAD) & $17(47.2)$ & $16(37.2)$ & $8(28.6)$ \\
\hline
\end{tabular}

Values are mean $\pm \mathrm{SD}$ or $n(\%)$. SAD, social anxiety disorder; GAD, generalized anxiety disorder.

interaction was significant $(p=0.04)$. Consistent with the prior analysis, COPE and RPT each showed significantly greater decreases in MPSS-SR scores compared to AMCG, regardless of PTSD diagnostic status (all $p$ values $<0.05)$. However, among participants with full PTSD, COPE also showed greater decreases relative to RPT (COPE-RPT $=-21.32,95 \% \mathrm{CI}-42.37$ to $-0.28, p=0.047$ ), whereas among participants with subthreshold PTSD, COPE was not significantly different from RPT ( $p=$ 0.92). In the follow-up model of CAPS scores, the 3-way interaction of group, PTSD status, and time was not significant $(p=0.26)$.

\section{Primary SUD Outcome}

There was a significant group-by-time interaction $(p<$ 0.001 ) in the model of past seven days of primary substance use (Fig. 3). Both treatments showed significant reductions in past seven days of primary substance use by the end-of-treatment session $(\mathrm{COPE}=-2.31,95 \% \mathrm{CI}$ -3.23 to $-1.39, p<0.001$; RP $=-3.28,95 \% \mathrm{CI}-4.03$ to 
Fig. 2. Model estimated means with $95 \%$ CIs of the Modified Posttraumatic Symptom Scale Self Report (MPSS-SR) of the total severity score at each weekly assessment during treatment. RZ, randomization visit; $\mathrm{S}$, session number.
Fig. 3. Model estimated means with $95 \%$ CIs of days of primary substance use reported in the Substance Use Inventory at each weekly assessment during treatment. $\mathrm{RZ}$, randomization visit; $\mathrm{S}$, session number.
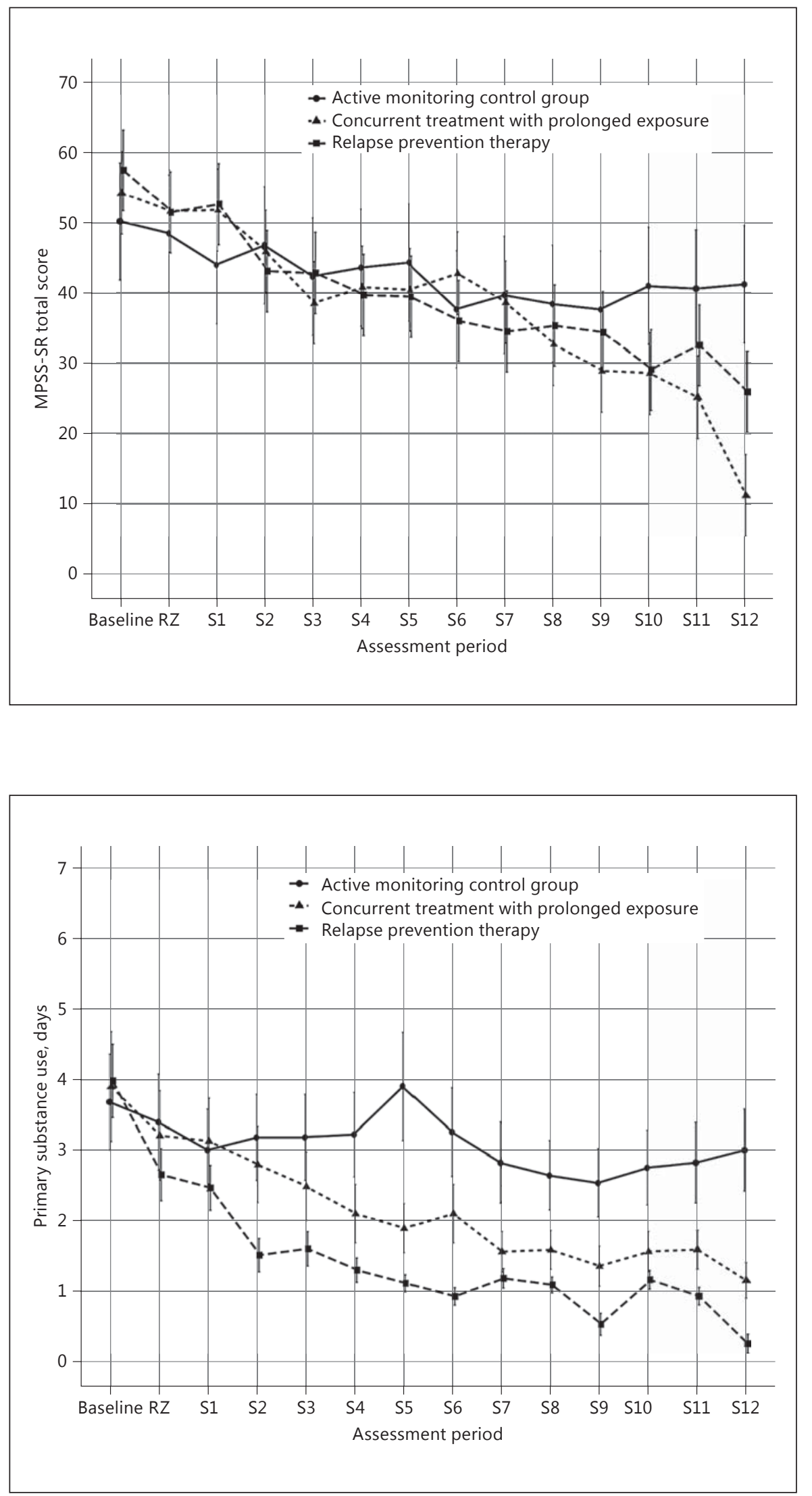
Table 2. Means and SDs of raw outcome data

\begin{tabular}{lccc}
\hline Outcome and time-point & COPE $(n=39)$ & RPT $(n=43)$ & AMCG $(n=28)$ \\
\hline Within-treatment self-report measures & & & \\
MPSS-SR total (past 7 days of PTSD severity) & & & \\
$\quad$ Baseline & $54.26(24.60)(n=39)$ & $57.49(24.33)(n=43)$ & $50.21(23.58)(n=28)$ \\
$\quad$ End-of-treatment & $19.40(17.70)(n=10)$ & $26.80(20.87)(n=10)$ & $40.00(28.10)(n=19)$ \\
SUI (past 7 days of primary substance use) & & & \\
$\quad$ Baseline & $3.90(2.69)(n=39)$ & $4.05(2.35)(n=43)$ & $3.79(2.27)(n=28)$ \\
$\quad$ End-of-treatment ${ }^{\text {a }}$ & $1.60(2.46)(n=10)$ & $0.40(0.52)(n=10)$ & $2.85(2.48)(n=20)$ \\
\hline Follow-up clinician administered measures & & & \\
CAPS (past 30 days of PTSD severity) & & & \\
Baseline & $55.38(16.40)(n=39)$ & $57.70(20.80)(n=43)$ & $46.39(11.07)(n=28)$ \\
$\quad$ Post-treatment ${ }^{b}$ & $37.63(23.76)(n=19)$ & $30.79(27.54)(n=24)$ & $41.89(24.52)(n=18)$ \\
1-Month follow-up & $29.50(27.88)(n=20)$ & $29.00(22.99)(n=29)$ & NA \\
2-Month follow-up & $29.77(26.14)(n=22)$ & $30.40(22.83)(n=25)$ & NA \\
3-Month follow-up & $28.40(23.09)(n=25)$ & $28.91(22.91)(n=23)$ & NA \\
ASI (past 30 days of primary substance use) & & & \\
$\quad$ Baseline & $18.23(10.55)(n=39)$ & $18.16(10.31)(n=42)$ & $21.79(8.36)(n=28)$ \\
Post-treatment & $11.60(10.30)(n=20)$ & $4.21(6.47)(n=24)$ & $13.74(9.74)(n=19)$ \\
1-Month follow-up & $8.65(11.34)(n=20)$ & $3.45(5.64)(n=29)$ & NA \\
2-Month follow-up & $10.82(11.85)(n=22)$ & $4.21(7.33)(n=24)$ & NA \\
3-Month follow-up & $8.08(9.95)(n=26)$ & $3.88(7.38)(n=24)$ & NA \\
\hline
\end{tabular}

Values are mean $\pm \mathrm{SD}$ or $n(\%) .{ }^{\text {a }}$ End-of-treatment assessments represent symptom levels over the last week or last month of treatment. ${ }^{\mathrm{b}}$ Post-treatment interviews were conducted 1 week after session 12 for the treatment groups, and include symptom levels over the last 3 weeks of treatment. For AMCG, post-treatment interviews were conducted during the 12th week visit.

$-2.53, p<0.001)$, whereas change in the AMCG was nonsignificant. The active treatment groups had significantly greater decreases relative to AMCG $(\mathrm{COPE}-\mathrm{AMCG}=$ $-0.97,95 \% \mathrm{CI}-1.72$ to $-0.22, p=0.01$; RPT-AMCG $=$ $-2.07,95 \%$ CI -2.92 to $-1.21, p<001)$. RPT also had significantly greater decrease relative to COPE (RPT$\mathrm{COPE}=-1.10,95 \% \mathrm{CI}-2.18$ to $-0.02, p=0.047)$.

Next, COPE and RPT were compared on past 30 days of primary substance use through follow-up. Both treatments showed significant reductions in number of days of primary substance use at the 1-month followup $(\mathrm{COPE}=-9.67,95 \% \mathrm{CI}-13.65$ to $-5.73, p<0.001$ $\mathrm{RPT}=-13.40,95 \% \mathrm{CI}-16.97$ to $-9.83, p<0.001)$ and 3 -month follow-up (COPE $=-10.45,95 \% \mathrm{CI}-14.27$ to $-6.63, p<0.001$; RPT $=-13.36,95 \% \mathrm{CI}-17.97$ to -8.74 , $p<0.001$ ), relative to baseline. There was no evidence of differential treatment effects, as indicated by the lack of a group-by-time interaction and the lack of between-group differences in primary substance use at follow-ups. Moreover, there were no within- or between-group differences in the change from the 1- to the 3-month follow-up. The end-of-treatment abstinence rates (past 7 days) were $12.8 \%(n=5)$ for COPE, $14 \%(n=6)$ for RPT, and $14.3 \%$

COPE for Co-Occurring PTSD and SUD $(n=4)$ for AMCG. By 3-month follow-up, abstinence rates (past 30 days) were $20.5 \%(n=8)$ for COPE and $27.9 \%(n=12)$ for RPT.

See Table 2 for means and SDs of raw outcome data at all time points by treatment condition.

\section{Discussion}

Our findings contribute further evidence for the efficacy of COPE, an integrated psychosocial treatment, for the common comorbidity of PTSD and SUD. We tested COPE against an evidence-based treatment for substance dependence as well as an AMCG. COPE and RPT produced clinically and statistically significant reductions in both self-reported and clinician-reported PTSD and SUD severity compared with AMCG. Although the difference between COPE and RPT was not significant in the complete sample, the subset of participants with full (vs. subthreshold) PTSD demonstrated significantly greater reduction of PTSD severity in COPE relative to RPT. In turn, RPT produced greater reductions of substance use relative to COPE at the end-of-treatment. Notably, PTSD

Psychother Psychosom 2017;86:150-161 157 
and SUD improvements in both active treatments were sustained, and no longer significantly different at follow-up.

Our study supports and builds upon COPE's first randomized trial conducted in an Australian sample of primarily past intravenous drug users with full PTSD. When compared to those who received only community-available SUD treatment, Mills et al. [3] found greater reductions in PTSD symptom severity for participants treated with COPE in conjunction with SUD treatment as usual. Participants in both groups decreased in substance dependence severity with no differences between groups at any follow-up. The present study adds to those findings by employing a strong, well-matched comparison treatment and shows COPE's utility in a wider, more ethnically diverse sample with significant polysubstance dependence and a range of trauma-related symptomatology. The positive treatment response in our sample suggests commonalities and consistency across populations of individuals with co-occurring PTSD and SUD, and the relevance of integrated approaches for PTSD + SUD care.

By demonstrating the effective delivery of a validated, exposure-based PTSD treatment, our findings represent an important step in the broadening of evidence-based treatment options for PTSD + SUD. Contrary to concerns regarding its tolerability, COPE produced significant improvements in PTSD and SUD severity that were sustained throughout follow-up. Indeed, for COPE, most of the PTSD-related gains were evidenced towards the end of treatment (by session 10), consistent with one of PE's hypothesized mechanism of change whereby multiple, cumulative sessions of memory processing (occurring in sessions 5 through 11 in this trial) consolidate and produce therapeutic results [42]. In addition to alleviating PTSD symptoms, COPE produced significant changes in substance use, suggesting that memory processing approaches can be implemented effectively among PTSD + SUD patients. Moreover, similar to the findings of Mills et al. [3], rates of abstinence were not high and did not differ between COPE and RPT. This information is critical in the light of the persistent call for abstinence prior to exposure-based trauma work [43]. In line with growing support for harm reduction and patient-centered approaches [44], our trial provides compelling evidence that exposure-based PTSD treatment can deliver significant PTSD relief in the context of substance abuse treatment, where abstinence has not yet been achieved or may not even be a goal. Although in need of replication, our finding regarding the enhanced benefit afforded by COPE to individuals with full PTSD highlights the advantage of pairing trauma memory processing with SUD treatments when targeting severe PTSD symptomatology in substance abuse populations.

Treatment drop-out rates, often cited as especially problematic in exposure-based interventions, did not differ between COPE and RPT. On average, participants receiving COPE attended approximately the same number of sessions $(n=6)$ as those in RPT $(n=7)$. Although vital for the recognition that exposure-based work does not differentially impact SUD treatment retention, the present study's overall high attrition rate highlights the reality confronted by PTSD + SUD clinicians and researchers. Client attrition in substance abuse programs remains one of the most intractable barriers to successful treatment outcomes [45]. The heightened challenge of engaging and retaining individuals with co-occurring PTSD and SUD in treatment is well documented and historically reflected in low sample sizes and high attrition rates of PTSD + SUD treatment trials [3, 19, 20, 46, 47]. The current study's retention rate closely resembles that of two recent trials of exposure-based PTSD treatment with PTSD + SUD individuals and many prior, non-exposurebased interventions. Mills et al. [3] reported a median of 5 out of 13 COPE sessions attended, and unlike the present study required no specific time frame for completion. In a combination trial with naloxone, participants completed on average approximately 6 out of $18 \mathrm{PE}$ sessions [20], reflecting only a third of the allotted sessions. A recent meta-analysis of psychological interventions for PTSD + SUD also stressed the difficulty of treatment retention [13]. In addition to the likelihood of treatment discontinuation afforded by a comorbid PTSD diagnoses, our study sample faced the compounded risks of multiple demographic and clinical characteristics associated with dropout: being a member of a target group, low social support, early onset of substance use, high SUD severity, and polydrug use [48-50]. As integrated practices for treating PTSD + SUD gain acceptance, arriving at a deeper understanding of the factors driving treatment discontinuation and designing adaptive interventions that increase adherence stand as essential next steps for PTSD + SUD research and care.

Several factors may have contributed to the lack of difference between trauma-focused and SUD-only treatment outcomes. Comparing dual disorder to single disorder treatments inherently poses certain methodological challenges. COPE, which integrates two individually efficacious treatments targeting separate disorders, was delivered in the same frequency and duration as employed by each single disorder treatment. As suggested by others 
[21], the dual disorder intervention may not have been adequately dosed for comparison to a single disorder treatment. In an uncontrolled study, COPE had an advantage among the subgroup of participants who completed at least three imaginal and four in vivo sessions [19]. In the present trial, under half (44\%) received at least this dose, potentially diffusing the impact of PE on PTSD. Moreover, when compared to a potent psychosocial treatment, sufficient dosage would be even more critical to achieving differential benefits. Nevertheless, findings from our moderator analyses provide preliminary evidence that the current dosage was differentially beneficial for individuals whose symptoms cross the threshold to full PTSD. Dosage constraints may also explain the endof-treatment RPT advantage in substance use reduction. With 90-min weekly sessions, RPT participants received double the amount of SUD-focused therapy relative to COPE participants, whose sessions were divided equally between PE and RPT. The lack of statistically significant differences between the two treatments at the end of the follow-up period may also be an artifact of low power. If the effect size differences between COPE and RPT were less than medium for the full sample (as we had estimated with a priori power analysis), our smaller than expected randomized sample size combined with attrition over the course of treatment may have reduced our chances of detecting significant differences between our active treatments. However, even if low power precluded detection of small effect sizes, these would not represent clinically meaningful differences. Importantly, within the treatment groups, there were statistically and clinically significant reductions across PTSD and SUD outcomes: the fact that both active treatments were so effective is another factor that may have contributed to the lack of observed differences between the groups in the amount of reduction. Nevertheless, this pattern is mirrored by the literature on PTSD treatments, substance abuse treatments, and psychotherapy in general: manualized models typically have been shown to be superior to the treatment as usual but do not outperform each other in direct comparisons [51, 52].

There were several other limitations as well. Because attrition was high, our findings may not generalize to individuals who dropped out; it is possible that those who remained in the treatment were more motivated than those who dropped out. Or those who dropped out may have seen early symptom improvement and no longer wanted treatment. Despite these limitations, the strengths of our study include the use of an active control group and time-matched treatments; a racially/ethnically diverse

COPE for Co-Occurring PTSD and SUD sample; multi-method (self-reported and clinician-assessed) and repeated assessments of PTSD and SUD; the broad range of trauma and substance use types; and welltrained and supervised clinicians with independent fidelity ratings. Contributing to the study's generalizability, the variety of substance use types and posttraumatic severity in this sample resemble the realities of community SUD programs, tasked with serving heterogeneous populations of substance users with varying degrees of dysfunction and levels of motivation to change. Although the sample size precluded analyses of outcomes by substance or trauma type, future research in these areas is crucial. Examining the impact of concurrent substance use on PTSD symptom change is particularly salient in the context of exposure therapy, as preclinical research demonstrates that acute and chronic cannabinoid [53, 54], alcohol [55], and cocaine [56] use may impact fear extinction - one of the putative therapeutic mechanisms of exposure. Future studies should examine the role of substance type on treatment response, which may help identify substance-related goals to be accomplished prior to exposure sessions to enhance learning and memory.

In conclusion, this study provides evidence that an integrated PTSD + SUD approach employing exposurebased techniques can be efficacious without exacerbation of substance use. Given the constraints of combining single disorder interventions, developing treatments for dysfunctions common across both disorders (e.g., affective and attentional disturbances) may prove critical. Research must also advance the science of treatment matching and the discovery of optimal treatment pathways, personalizing approaches for this common yet heterogeneous subpopulation.

\section{Disclosure Statement}

Drs. L.M. Ruglass, T. Lopez-Castro, D.A. Hien and Mr. S. Papini declare they have no conflicts of interest regarding the publication of this paper. Drs. T. Killeen and S. Back report they receive royalties from Oxford University Press for a treatment manual they wrote on utilizing COPE.

\section{Funding Source}

This study was supported by a grant from the National Institute on Drug Abuse (R01DA10843; PI: Denise A. Hien, PhD). 


\section{References}

1 Grant BF, Stinson FS, Dawson DA, Chou SP, Dufour MC, Compton W, Pickering RP, Kaplan K: Prevalence and co-occurrence of substance use disorders and independent mood and anxiety disorders: results from the national epidemiologic survey on alcohol and related conditions. Arch Gen Psychiatry 2004; 61:807-816.

2 Jacobsen LK, Southwick SM, Kosten TR: Substance use disorders in patients with posttraumatic stress disorder: a review of the literature. Am J Psychiatry 2001;158:1184-1190.

-3 Mills KL, Teesson M, Back SE, Brady KT, Baker AL, Hopwood S, Sannibale C, Barrett EL, Merz S, Rosenfeld J, et al: Integrated exposure-based therapy for co-occurring posttraumatic stress disorder and substance dependence: a randomized controlled trial. JAMA 2012;308:690-699.

$\checkmark 4$ Reynolds M, Hinchliffe K, Asamoah V, Kouimtsidis C: Trauma and post-traumatic stress disorder in a drug treatment community service. Psychiatrist 2011;35:256-260.

$\checkmark 5$ Najavits LM, Hien D: Helping vulnerable populations: a comprehensive review of the treatment outcome literature on substance use disorder and PTSD. J Clin Psychol 2013; 69:433-479.

6 Schäfer I, Najavits LM: Clinical challenges in the treatment of patients with posttraumatic stress disorder and substance abuse. Curr Opin Psychiatry 2007;20:614-618.

7 Ruglass LM, Lopez-Castro T, Cheref S, Papini S, Hien DA: At the crossroads: the intersection of substance use disorders, anxiety disorders, and posttraumatic stress disorder. Curr Psychiatry Rep 2014;16:1-9.

8 Difede J, Cukor J, Wyka K, Olden M, Hoffman H, Lee FS, Altemus M: D-cycloserine augmentation of exposure therapy for posttraumatic stress disorder: a pilot randomized clinical trial. Neuropsychopharmacology 2014;39:1052-1058.

9 Foa EB: Prolonged exposure therapy: past, present, and future. Depress Anxiety 2011;28: 1043-1047.

10 Gold PB, Brady KT: Evidence-based treatments for substance use disorders. Focus (Madison) 2003;1:115-122.

11 Pilkey D, Steinberg H, Martino S: Evidencebased treatments for substance use disorders; in Kaye AD, Vadivelu N, Urman R (eds): Substance Abuse. New York, Springer, 2015, pp 209-227.

12 van Dam D, Vedel E, Ehring T, Emmelkamp PM: Psychological treatments for concurrent posttraumatic stress disorder and substance use disorder: a systematic review. Clin Psychol Rev 2012;32:202-214.

13 Roberts NP, Roberts PA, Jones N, Bisson JI: Psychological interventions for post-traumatic stress disorder and comorbid substance use disorder: a systematic review and metaanalysis. Clin Psychol Rev 2015;38:25-38.
14 Foa E, Hembree E, Rothbaum BO: Prolonged Exposure Therapy for PTSD: Emotional Processing of Traumatic Experiences Therapist Guide, 2007.

-15 Powers MB, Halpern JM, Ferenschak MP, Gillihan SJ, Foa EB: A meta-analytic review of prolonged exposure for posttraumatic stress disorder. Clin Psychol Rev 2010;30:635-641.

16 Back SE: Toward an improved model of treating co-occurring PTSD and substance use disorders. Am J Psychiatry 2010;167:11-13.

17 Mills K: Treatment of comorbid substance dependence and posttraumatic stress disorder. JAMA 2013;310:482-483.

18 Back SE, Killeen T, Foa EB, Santa Ana EJ, Gros DF, Brady KT: Use of an integrated therapy with prolonged exposure to treat PTSD and comorbid alcohol dependence in an Iraq veteran. Am J Psychiatry 2012;169:688-691.

19 Brady KT, Dansky BS, Back SE, Foa EB, Carroll KM: Exposure therapy in the treatment of PTSD among cocaine-dependent individuals: preliminary findings. J Subst Abuse Treat 2001;21:47-54

20 Foa EB, Yusko DA, McLean CP, Suvak MK, Bux DA Jr, Oslin D, O’Brien CP, Imms P, Riggs DS, Volpicelli J: Concurrent naltrexone and prolonged exposure therapy for patients with comorbid alcohol dependence and PTSD: a randomized clinical trial. JAMA 2013;310:488-495.

21 Sannibale C, Teesson M, Creamer M, Sitharthan T, Bryant RA, Sutherland K, Taylor K, Bostock-Matusko D, Visser A, Peek-O'Leary M: Randomized controlled trial of cognitive behaviour therapy for comorbid post-traumatic stress disorder and alcohol use disorders. Addiction 2013;108:1397-1410.

22 Marlatt GA, Donovan DM: Relapse Prevention: Maintenance Strategies in the Treatment of Addictive Behaviors. New York, Guilford Press, 2007.

23 American Psychiatric Association: Diagnostic and Statistical Manual of Mental Disorders, ed 4. Washington, American Psychiatric Association, 2000.

-24 Grubaugh AL, Magruder KM, Waldrop AE, Elhai JD, Knapp RG, Frueh BC: Subthreshold PTSD in primary care: prevalence, psychiatric disorders, healthcare use, and functional status. J Nerv Ment Dis 2005;193:658-664.

25 Zlotnick C, Franklin CL, Zimmerman M: Does "subthreshold" posttraumatic stress disorder have any clinical relevance? Compr Psychiatry 2002;43:413-419.

26 Pietrzak RH, Goldstein RB, Southwick SM, Grant BF: Prevalence and Axis I comorbidity of full and partial posttraumatic stress disorder in the United States: results from Wave 2 of the National Epidemiologic Survey on Alcohol and Related Conditions. J Anxiety Disord 2011;25:456-465.
Pietrzak RH, Goldstein MB, Malley JC, Johnson DC, Southwick SM: Subsyndromal posttraumatic stress disorder is associated with health and psychosocial difficulties in veterans of operations enduring freedom and Iraqi freedom. Depress Anxiety 2009;26: 739-744.

28 Jin H, Rourke SB, Patterson TL, Taylor MJ, Grant I: Predictors of relapse in long-term abstinent alcoholics. J Stud Alcohol 1998;59: 640-646.

29 Bradizza CM, Stasiewicz PR, Paas ND: Relapse to alcohol and drug use among individuals diagnosed with co-occurring mental health and substance use disorders: a review. Clin Psychol Rev 2006;26:162-178.

30 Back SE, Foa EB, Killeen TK, Mills KL, Teesson $\mathrm{M}$, Cotton BD, Carroll KM, Brady KT: Concurrent Treatment of PTSD and Substance Use Disorders Using Prolonged Exposure (COPE): Therapist Guide. New York, Oxford University Press, 2014.

31 Carroll KM: A Cognitive-Behavioral Approach: Treating Cocaine Addiction. Rockville, National Institute on Drug Abuse, 1998.

32 Carroll KM, Kadden RM, Donovan DM, Zweben A, Rounsaville BJ: Implementing treatment and protecting the validity of the independent variable in treatment matching studies. J Stud Alcohol Suppl 1994;149-155.

33 First MB, Spitzer RL, Gibbon M, Williams JB: Structured Clinical Interview for DSM-IVTR Axis I Disorders, Research Version, Patient Edition with Psychotic Screen. New York, Biometrics Research, New York State Psychiatric Institute, 2002.

34 Blake DD, Weathers FW, Nagy LM, Kaloupek DG, Gusman FD, Charney DS, Keane TM: The development of a clinician-administered PTSD scale. J Trauma Stress 1995;8:75-90.

35 Falsetti SA, Resnick HS, Resick PA, Kilpatrick DG: The Modified PTSD Symptom Scale: a brief self-report measure of posttraumatic stress disorder. Behav Ther 1993;16:161-162.

36 Ruglass LM, Papini S, Trub L, Hien DA: Psychometric properties of the modified posttraumatic stress disorder symptom scale among women with posttraumatic stress disorder and substance use disorders receiving outpatient group treatments. J Trauma Stress Disord Treat 2014;4:1-7.

37 Cacciola JS, Alterman AI, McLellan AT, Lin YT, Lynch KG: Initial evidence for the reliability and validity of a "Lite" version of the Addiction Severity Index. Drug Alcohol Depend 2007;87:297-302.

38 Weiss RD, Hufford C, Najavits LM: Weekly Substance Use Inventory. Boston, Harvard Medical School, 1995.

39 Diggle P, Heagerty P, Liang K-Y, Zeger S: Analysis of Longitudinal Data. New York, Oxford University Press, 2002. 
40 Najavits LM, Weiss RD, Shaw SR, Muenz LR: "Seeking safety": outcome of a new cognitivebehavioral psychotherapy for women with posttraumatic stress disorder and substance dependence. J Trauma Stress 1998;11:437456.

41 Coffey SF, Stasiewicz PR, Hughes PM, Brimo ML: Trauma-focused imaginal exposure for individuals with comorbid posttraumatic stress disorder and alcohol dependence: revealing mechanisms of alcohol craving in a cue reactivity paradigm. Psychol Addict Behav 2006;20:425-435.

42 Foa EB, Cahill S: Psychological therapies: emotional processing; in Smelser N, Bates $\mathrm{P}$ (eds): International Encyclopedia of the Social and Behavioral Sciences. Oxford, Elsevier, 2001, pp 12363-12369.

43 Najavits LM, Norman SB, Kivlahan D, Kosten TR: Improving PTSD/substance abuse treatment in the VA: a survey of providers. Am Addict 2010;19:257-263.

-44 Lozano BE, Gros DF, Killeen T, Jaconis M, Beylotte FM 3rd, Boyd S, Back SE: To reduce or abstain? Substance use goals in the treatment of veterans with substance use disorders and comorbid PTSD. Am J Addict 2015;24: 578-581.

45 Loveland D, Driscoll H: Examining attrition rates at one specialty addiction treatment provider in the United States: a case study using a retrospective chart review. Subst Abuse Treat Prev Policy 2014;9:41.
46 Najavits LM, Weiss RD, Shaw SR, Muenz LR: "Seeking safety": outcome of a new cognitivebehavioral psychotherapy for women with posttraumatic stress disorder and substance dependence. J Trauma Stress 1998;11:437456.

47 Hien DA, Wells EA, Jiang H, Suarez-Morales L, Campbell AN, Cohen LR, Miele GM, Killeen T, Brigham GS, Zhang Y, Hansen C, Hodgkins C, Hatch-Maillette M, Brown C, Kulaga A, Kristman-Valente A, Chu M, Sage R, Robinson JA, Liu D, Nunes EV: Multisite randomized trial of behavioral interventions for women with co-occurring PTSD and substance use disorders. J Consult Clin Psychol 2009;77:607-619.

48 Veach LJ, Remley TP Jr, Kippers SM, Sorg JD: Retention predictors related to intensive outpatient programs for substance use disorders. Am J Drug Alcohol Abuse 2000;26:417-428.

49 Ball SA, Carroll KM, Canning-Ball M, Rounsaville BJ: Reasons for dropout from drug abuse treatment: symptoms, personality, and motivation. Addict Behav 2006;31:320-330.

50 Fishman J, Reynolds T, Riedel E: A retrospective investigation of an intensive outpatient substance abuse treatment program. Am J Drug Alcohol Abuse 1999;25:185-196.
Benish SG, Imel ZE, Wampold BE: The relative efficacy of bona fide psychotherapies for treating post-traumatic stress disorder: a meta-analysis of direct comparisons. Clin Psychol Rev 2008;28:746-758.

52 Bisson JI, Roberts NP, Andrew M, Cooper R, Lewis C: Psychological therapies for chronic post-traumatic stress disorder (PTSD) in adults. Cochrane Database Syst Rev 2013; 12:CD003388.

53 Papini S, Sullivan GM, Hien DA, Shvil E, Neria Y: Toward a translational approach to targeting the endocannabinoid system in posttraumatic stress disorder: a critical review of preclinical research. Biol Psychol 2015;104: 8-18.

54 Papini S, Ruglass LM, Lopez-Castro T, Powers MB, Smits JA, Hien DA: Chronic cannabis use is associated with impaired fear extinction in humans. J Abnorm Psychol 2016;126:117124.

55 Holmes A, Fitzgerald PJ, MacPherson KP, DeBrouse L, Colacicco G, Flynn SM, Masneuf S, Pleil KE, Li C, Marcinkiewcz CA, et al: Chronic alcohol remodels prefrontal neurons and disrupts NMDAR-mediated fear extinction encoding. Nat Neurosci 2012;15:13591361.

-56 Burke KA, Franz TM, Gugsa N, Schoenbaum G: Prior cocaine exposure disrupts extinction of fear conditioning. Learn Mem 2006;13: 416-421. 